\title{
Exploration of cytoplasmic inheritance as a contributor to maternal effects in Welsh Mountain sheep
}

\author{
Tracey PRITChARD*, Christine CAHAlan, Ioan AP DEWI \\ School of the Environment and Natural Resources, University of Wales, Bangor, \\ LL57 2UW, UK
}

(Received 17 April 2007; accepted 22 October 2007)

\begin{abstract}
Cytoplasmic effects were investigated using a dataset comprising three breeding groups of Welsh Mountain sheep. The influences of cytoplasmic effects were investigated by comparing animal models with and without a random term representing cytoplasmic effects. The models were applied to the eight-week weight, scan weight (mean 152 days) and ultrasonically scanned muscle and fat depth. The animal model included the random effects of animals and the maternal additive genetic, maternal permanent environmental and maternal common environmental effects. In total there were $24569,10509,8389,8369$ records for the eight-week weight, scan weight, muscle depth and fat depth respectively. Four subsets were further analysed containing maternal lines with at least five, ten, fifteen and twenty animals/line. There was no evidence of cytoplasmic effects on eight-week weight and muscle depth. Cytoplasmic effects contributed 1-2\% of phenotypic variance for scan-weight and fat depth, but the effect was generally non-significant $(\mathrm{P}>0.05)$. As the number of animals per maternal line increased, the magnitude of cytoplasmic effects also increased for these traits. Direct heritability estimates for the eight-week weight, scan weight, muscle depth and fat depth using the full dataset were 0.18 , $0.25,0.24$, and 0.21 respectively.
\end{abstract}

cytoplasmic inheritance / model / maternal effect / sheep

\section{INTRODUCTION}

Wagner [25] discussed the importance of mitochondria in animal breeding, pointing out that they could be responsible for genetic variation in cytoplasmic effects since they contained maternally inherited DNA. Although a few studies have reported possible paternal inheritance [26], in mammals, mtDNA on the main is inherited from the mother because sperm-derived mitochondria are degraded during embryogenesis [7]. Males receive cytoplasmic material from their dams, but do not transmit it to their offspring. Hence, apart from

*Corresponding author: pritchard.tc@gmail.com

Article published by EDP Sciences and available at http://www.gse-journal.org or http://dx.doi.org/10.1051/gse:2008005 
mutations, animals of a maternal line should have identical mtDNA, and theoretically, the component of variance due to mtDNA can be estimated [13].

It has been suggested that cytoplasmic effects could exist in sheep and some studies have investigated the association between mtDNA polymorphisms with phenotypic variation in sheep, but so far these have failed to show any evidence of cytoplasmic effects. Maniatis and Pollott [8] found no evidence of cytoplasmic effects, when included in a full animal model, for eight-week weight, scanning weight or ultrasonic measurements of muscle and fat depth in Suffolk lambs. Similarly, in Columbia [6], Targee [22], Rambouillet [19] and Polpay [23] breeds, cytoplasmic effects appeared to be unimportant for the traits birth weight, weaning weight, fleece weight and the number of lambs born. Hence, these authors considered that it was not necessary to include such effects in genetic evaluation models.

The implications of cytoplasmic genetic effects affecting performance are that some maternal lines might be particularly valued, due to the inheritance of cytoplasmic elements from the dam [21]. Schutz et al. [16] suggested that a sire's estimated breeding value, based upon pedigree, might be biased if the dam's contribution is not adjusted for mitochondrial effects. Current breeding evaluations do not include the effect of cytoplasmic inheritance through the maternal line. The aim of this study was to estimate the contribution of maternal lines to weight and ultrasonically-scanned traits of the Welsh Mountain breed in order to determine whether ignoring cytoplasmic effects is appropriate to estimate breeding values.

\section{MATERIALS AND METHODS}

\subsection{Description of data}

Data from three breeding groups of the Welsh Mountain breed, CAMDA, CAMP, and Llysfasi, were used to study the effect of cytoplasmic inheritance. A data file and a pedigree file were obtained for each breeding group from the Meat and Livestock Commission. The data was edited and from an initial 28830 animals, 24569 remained in the dataset. Uncommon birth rearing types, one-year-old dams, and trait records that were more than three standard deviations from the trait mean were removed from the dataset. There were only two animals with scanning measurements in 1997, so they were removed from the dataset. The traits analysed were eight-week weight (EWW), weight at scanning (SW) measured at 20/21 weeks, ultrasonic muscle depth (MD), and ultrasonic fat depth (FD). Ultrasonic scanning measurements of muscle and fat depths were taken from the third lumbar vertebra [18]. Age at scanning was used as a covariate for SW, MD and FD and the mean age was 152 days. 
There were 452 sires and 9883 dams from years 1985 to 2004 from 40 flocks. All had records for EWW, but there were fewer records for SW, $\mathrm{MD}$, and FD (Tab. I). In the pedigree file, there were 12 generations and the oldest dam was born in 1969. The number of offspring per dam ranged from one to 14, and averaged 2.49 lambs. There were 18029 litter groups (animals with the same dam of the same year). The number of lambs per litter group ranged from one to two, and average litter size was 1.36 lambs.

\subsection{Data analysis and models used}

The investigation of cytoplasmic effects required assigning animals to maternal lines by identifying foundation females. Each foundation female was assumed to be unrelated to the other foundation dams. Foundation females were regarded as a cytoplasmic source and given individual codes. All descendants were given the same code as the foundation females, since these animals were assumed to have the same mtDNA genotype within the line (i.e. it was assumed that no mutation of mtDNA had taken place). It was observed that some lines had very few animals, for instance lines consisting of a foundation dam with only one descendant. These lines with few animals might be expected to bias an analysis to determine the importance of cytoplasmic effects. Therefore, four subsets of the full dataset, with a minimum of 5, 10, 15, and 20 animals per maternal line, were also analysed. Increasing the minimum number of animals allowed in each line caused a successive reduction in the size of the datasets (Tab. I).

The full dataset and four subsets were analysed with ASReml [5] using a univariate animal model with and without maternal line as a random effect. The fixed effects were dam age, year of birth, sex, birth rearing type and flock. Year of birth, sex, birth rearing type and flock were combined as one effect, and this accounted for all interactions between the fixed effects. Animal age was included as a covariate as the age of the animal, in days, at the time of weighing and ultrasonic scanning for scan weight, muscle depth and fat depth.

The random effects were the direct additive genetic effect, maternal additive genetic effect, maternal permanent environmental effect, and maternal common environmental effects. The model was analysed with and without maternal line as a random effect, as shown in equation (1) below:

$$
Y_{i j k l m n o}=\mu+a_{i}+b_{j k l m}+c_{n}+z_{n}+x_{o}+w_{o}+v_{o l}+u_{o}+e_{i j k l m n o}
$$

where $\mu=$ is the overall mean of eight-week weight, scan-weight, muscle depth or fat depth; $a_{i}=$ fixed effect of rearing dam age (4 age classes: $2,3,4-6,7+$ ); 
Table I. Numbers of records, sires, dams and maternal lines for eight-week weight (EWW), scan weight (SW), muscle depth (MD) and fat depth (FD) of Welsh Mountain sheep. The values shown are for the full dataset and for subsets containing maternal lines with at least five and 20 animals/line.

\begin{tabular}{lccccc}
\hline Trait & Dataset & Count & Nb. of sires & Nb. of dams & Nb. of maternal lines \\
\hline EWW & All & 24569 & 452 & 9883 & 5302 \\
$(\mathrm{~kg})$ & $5+$ & 16586 & 380 & 4989 & 655 \\
& $20+$ & 12689 & 307 & 3721 & 219 \\
\hline SW & All & 10509 & 241 & 5724 & 3932 \\
$(\mathrm{~kg})$ & $5+$ & 5179 & 169 & 2185 & 527 \\
& $20+$ & 3210 & 105 & 1379 & 187 \\
\hline MD & All & 8389 & 235 & 4872 & 3352 \\
$(\mathrm{~mm})$ & $5+$ & 4269 & 167 & 1961 & 518 \\
& $20+$ & 2437 & 105 & 1172 & 180 \\
\hline FD & All & 8369 & 235 & 4859 & 3339 \\
$(\mathrm{~mm})$ & $5+$ & 4267 & 167 & 1960 & 517 \\
& $20+$ & 2437 & 105 & 1172 & 180 \\
\hline
\end{tabular}

$b_{j k l m}$ contemporary group of $j^{\text {th }}$ sex (male/female), $k^{\text {th }}$ birth rearing type ( 3 classes: single born-single reared, twin born-single reared, twin born-twin reared), $l^{\text {th }}$ year of birth (20 classes: 1985 to 2004), and $m^{\text {th }}$ flock (1-40); $c_{n}=$ age at scanning as a covariate of the animal $n$ (not needed for eight-week weight); $z_{n}=$ random effect of animal $n ; x_{o}=$ the random maternal additive genetic effect of the $o^{\text {th }}$ dam; $w_{o}=$ the random maternal permanent environmental effect of the $o^{\text {th }}$ dam; $v_{o l}=$ the random maternal common environmental effect of the $o^{\text {th }}$ dam in the $l^{\text {th }}$ year; $u_{o}=$ cytoplasmic genetic effect of the $o^{\text {th }}$ dam; $e_{i j k l m n o}=$ random environmental effect.

The significance of the cytoplasmic effect was determined using a likelihood ratio test $(\mathrm{P}=0.05)$, a method widely used to compare models in these types of analyses $[2,8,11]$, and was considered to have a significant influence when its addition in Model 2 caused a significant increase in $-2 * \log \mathrm{L}$, compared to Model 1 without the effect. The test statistic follows a 50:50 mixture of chisquared distributions with respectively 0 and 1 degree of freedom, therefore at a significance level of 0.05 a critical value of 2.79 was used. Whilst increasing the number of parameters may increase the goodness of fit, there is a danger of overparameterisation [17]. Therefore, to discourage overfitting, the choice of model was also judged by Akaike's Information Criterion AIC $=-2 \log \mathrm{L}+$ $2 k$ [24] and the Bayesian Information Criterion BIC $=-2 \log L+k \ln (n)[1,17]$ where $\mathrm{k}=$ number of independent estimated parameters, and $\mathrm{n}=$ total number of observations. The preferred model chosen by AIC and BIC is that with the lowest value. 
Table II. Variance components and genetic parameters for eight-week weight, scan weight, muscle depth and fat depth for the full dataset excluding cytoplasmic effect.

\begin{tabular}{lcccc}
\hline Model & EWW & SW & MD & FD \\
\hline$\sigma_{\mathrm{a}}^{2}$ & 1.21 & 2.87 & 1.12 & 0.14 \\
$\sigma_{\mathrm{m}}^{2}$ & 0.36 & 0.55 & 0.10 & 0.03 \\
$\sigma_{\mathrm{pe}}^{2}$ & 0.41 & 0.84 & 0.16 & 0.00 \\
$\sigma_{\mathrm{ce}}^{2}$ & 1.57 & 1.58 & 0.39 & 0.10 \\
$\sigma_{\mathrm{e}}^{2}$ & 3.01 & 5.69 & 2.85 & 0.37 \\
$\sigma_{\mathrm{p}}^{2}$ & $6.56 \pm 0.07$ & $11.51 \pm 0.20$ & $4.62 \pm 0.09$ & $0.61 \pm 0.12$ \\
$\mathrm{~h}^{2}$ & $0.18 \pm 0.02$ & $0.25 \pm 0.04$ & $0.24 \pm 0.04$ & $0.21 \pm 0.04$ \\
$\mathrm{~m}^{2}$ & $0.06 \pm 0.01$ & $0.05 \pm 0.02$ & $0.02 \pm 0.02$ & $0.05 \pm 0.02$ \\
$\mathrm{pe}^{2}$ & $0.06 \pm 0.01$ & $0.07 \pm 0.02$ & $0.03 \pm 0.02$ & 0.00 \\
$\mathrm{ce}^{2}$ & $0.24 \pm 0.01$ & $0.14 \pm 0.02$ & $0.09 \pm 0.03$ & $0.16 \pm 0.03$ \\
\hline
\end{tabular}

$\sigma_{\mathrm{a}}^{2}$ : direct additive effect; $\sigma_{\mathrm{m}}^{2}$ : maternal additive genetic variance; $\sigma_{\mathrm{pe}}^{2}:$ maternal permanent environmental variance; $\sigma_{\mathrm{ce}}^{2}$ : common environmental variance; $\sigma_{\mathrm{e}}^{2}$ : error variance; $\sigma_{\mathrm{p}}^{2}$ : phenotypic variance; $\mathrm{h}^{2}$ : direct heritability; $\mathrm{m}^{2}:$ maternal heritability; $\mathrm{pe}^{2}$ : maternal permanent environmental variance expressed as a proportion of phenotypic variance; $\mathrm{ce}^{2}$ : maternal common environmental variance expressed as a proportion of the phenotypic variance.

\section{RESULTS}

Genetic parameter estimates for eight-week weight (EWW), scan weight (SW) and ultrasonically measured traits muscle depth (MD) and fat depth (FD) are presented in Table II using the full dataset without cytoplasmic effect. For EWW and MD the inclusion of cytoplasmic inheritance as a random effect in the model made no difference to the $\log L$ values. In each analysis for MD the cytoplasmic effect was fixed at a boundary, because its contribution to phenotypic variance was so small. For all subsets of data for SW and $\mathrm{FD}$, the inclusion of cytoplasmic effects resulted in more positive $\log \mathrm{L}$ values (Tabs. III and IV). However, only for the dataset containing all animals for SW was there a significant difference $(\mathrm{P}>0.05)$ in $\log \mathrm{L}$ value between the two models. For both SW and FD, cytoplasmic effects ranged from 1-2\% of the phenotypic variance and increased as the cut-off point for the number of animals per maternal line increased (or with decreasing size of the dataset). The phenotypic variances and residual errors remained approximately the same in both models, which meant that, for SW and FD, the inclusion of cytoplasmic effect caused slight decreases in direct and maternal heritability. Comparison of datasets showed that increasing the number of animals per maternal line 
Table III. Variance components and genetic parameters for scan weight with models that exclude (Model 1) or include (Model 2) cytoplasmic effects for the full dataset and for subsets containing maternal lines with at least five and 20 animals/line.

\begin{tabular}{|c|c|c|c|c|c|c|}
\hline \multirow{2}{*}{ Model } & \multicolumn{2}{|c|}{$1+$} & \multicolumn{2}{|c|}{$5+$} & \multicolumn{2}{|c|}{$20+$} \\
\hline & 1 & 2 & 1 & 2 & 1 & 2 \\
\hline$\sigma_{\mathrm{a}}^{2}$ & 2.87 & 2.77 & 2.32 & 2.24 & 2.80 & 2.78 \\
\hline$\sigma_{\mathrm{m}}^{2}$ & 0.55 & 0.43 & 0.70 & 0.54 & 0.73 & 0.47 \\
\hline$\sigma_{\mathrm{pe}}^{2}$ & 0.84 & 0.84 & 0.99 & 1.01 & 0.80 & 0.86 \\
\hline$\sigma_{\mathrm{ce}}^{2}$ & 1.58 & 1.57 & 1.24 & 1.24 & 1.35 & 1.35 \\
\hline$\sigma_{\text {cy }}^{2}$ & - & 0.16 & - & 0.17 & - & 0.22 \\
\hline$\sigma_{\mathrm{e}}^{2}$ & 5.69 & 5.72 & 6.15 & 6.19 & 6.20 & 6.20 \\
\hline$\sigma_{\mathrm{p}}^{2}$ & $11.51 \pm 0.20$ & $11.49 \pm 0.20$ & $11.41 \pm 0.28$ & $11.39 \pm 0.28$ & $11.88 \pm 0.37$ & $11.88 \pm 0.37$ \\
\hline$h^{2}$ & $0.25 \pm 0.04$ & $0.24 \pm 0.04$ & $0.20 \pm 0.05$ & $0.20 \pm 0.04$ & $0.24 \pm 0.06$ & $0.23 \pm 0.06$ \\
\hline $\mathrm{m}^{2}$ & $0.05 \pm 0.02$ & $0.04 \pm 0.02$ & $0.06 \pm 0.03$ & $0.05 \pm 0.03$ & $0.06 \pm 0.04$ & $0.04 \pm 0.03$ \\
\hline $\mathrm{pe}^{2}$ & $0.07 \pm 0.02$ & $0.07 \pm 0.02$ & $0.09 \pm 0.03$ & $0.09 \pm 0.03$ & $0.07 \pm 0.03$ & $0.07 \pm 0.03$ \\
\hline $\mathrm{ce}^{2}$ & $0.14 \pm 0.02$ & $0.14 \pm 0.02$ & $0.11 \pm 0.03$ & $0.11 \pm 0.03$ & $0.11 \pm 0.03$ & $0.11 \pm 0.03$ \\
\hline$c y^{2}$ & - & $0.01 \pm 0.01$ & - & $0.02 \pm 0.01$ & - & $0.02 \pm 0.01$ \\
\hline$-2 * \log L$ & 34454 & 34434.6 & 16871.9 & 16869.76 & 10747 & 10744.4 \\
\hline AIC & 34462 & 34444.6 & 16879.9 & 16879.76 & 10755 & 10754.4 \\
\hline BIC & 34495.79 & 34486.83 & 16910.83 & 16918.43 & 10784.13 & 10790.81 \\
\hline
\end{tabular}

$\sigma_{\mathrm{a}}^{2}$ : direct additive effect; $\sigma_{\mathrm{m}}^{2}$ : maternal additive genetic variance; $\sigma_{\mathrm{pe}}^{2}:$ maternal permanent environmental variance; $\sigma_{\text {ce }}^{2}$ : common environmental variance; $\sigma_{\text {cy }}^{2}$ : cytoplasmic genetic variance; $\sigma_{\mathrm{e}}^{2}$ : error variance; $\sigma_{\mathrm{p}}^{2}$ : phenotypic variance; $\mathrm{h}^{2}$ : direct heritability; $\mathrm{m}^{2}:$ maternal heritability; $\mathrm{pe}^{2}$ : maternal permanent environmental variance expressed as a proportion of phenotypic variance; $\mathrm{ce}^{2}$ : maternal common environmental variance expressed as a proportion of the phenotypic variance; $\mathrm{cy}^{2}$ : cytoplasmic genetic variance expressed as a proportion of phenotypic variance; $-2 * \log L$ : $\log$ likelihood ratio test: AIC: Akaike Information Criterion; BIC: Bayesian Information Criterion. Value in bold shows the chosen model according to test statistic.

resulted in increases or decreases in the variance components and phenotypic variances (Tabs. III and IV).

Testing models with AIC and BIC gave similar results as comparing differences between $-2 * \log \mathrm{L}$. With the use of AIC more models with cytoplasmic effect were chosen, however, the differences between the two models were very small. BIC penalises free parameters more strongly than AIC and by testing models by this statistic only one dataset, containing all animals for SW, was the model with cytoplasmic effect chosen.

\section{DISCUSSION}

Direct heritability estimates were similar to those of Ap Dewi et al. [2] obtained from the CAMDA Welsh Mountain flock. Maternal additive genetic, 
Table IV. Variance components and genetic parameters for fat depth with models that exclude (Model 1) or include (Model 2) cytoplasmic effects for the full dataset and for subsets containing maternal lines with at least five and 20 animals/line.

\begin{tabular}{|c|c|c|c|c|c|c|}
\hline \multirow{2}{*}{ Model } & \multicolumn{2}{|c|}{$1+$} & \multicolumn{2}{|c|}{$5+$} & \multicolumn{2}{|c|}{$20+$} \\
\hline & 1 & 2 & 1 & 2 & 1 & 2 \\
\hline$\sigma_{\mathrm{a}}^{2}$ & 0.14 & 0.13 & 0.15 & 0.14 & 0.10 & 0.09 \\
\hline$\sigma_{\mathrm{m}}^{2}$ & 0.03 & 0.02 & 0.03 & 0.02 & 0.04 & 0.03 \\
\hline$\sigma_{\mathrm{pe}}^{2}$ & 0.00 & 0.00 & 0.00 & 0.01 & 0.00 & 0.00 \\
\hline$\sigma_{\mathrm{ce}}^{2}$ & 0.10 & 0.10 & 0.08 & 0.08 & 0.11 & 0.11 \\
\hline$\sigma_{\mathrm{cy}}^{2}$ & - & 0.01 & - & 0.01 & - & 0.01 \\
\hline$\sigma_{\mathrm{e}}^{2}$ & 0.37 & 0.38 & 0.38 & 0.38 & 0.40 & 0.40 \\
\hline$\sigma_{\mathrm{p}}^{2}$ & $0.61 \pm 0.12$ & $0.64 \pm 0.01$ & $0.65 \pm 0.02$ & $0.64 \pm 0.02$ & $0.66 \pm 0.02$ & $0.66 \pm 0.02$ \\
\hline$h^{2}$ & $0.21 \pm 0.04$ & $0.20 \pm 0.04$ & $0.24 \pm 0.05$ & $0.22 \pm 0.05$ & $0.16 \pm 0.06$ & $0.14 \pm 0.05$ \\
\hline $\mathrm{m}^{2}$ & $0.05 \pm 0.02$ & $0.04 \pm 0.02$ & $0.04 \pm 0.02$ & $0.03 \pm 0.02$ & $0.07 \pm 0.03$ & $0.05 \pm 0.03$ \\
\hline $\mathrm{pe}^{2}$ & 0.00 & 0.00 & $0.01 \pm 0.02$ & $0.01 \pm 0.02$ & 0.00 & 0.00 \\
\hline $\mathrm{ce}^{2}$ & $0.16 \pm 0.03$ & $0.16 \pm 0.03$ & $0.13 \pm 0.04$ & $0.13 \pm 0.04$ & $0.17 \pm 0.05$ & $0.18 \pm 0.05$ \\
\hline $\mathrm{cy}^{2}$ & - & $0.01 \pm 0.01$ & - & $0.02 \pm 0.01$ & - & $0.02 \pm 0.01$ \\
\hline$-2 * \log L$ & 4925.68 & 4923.74 & 2409.54 & 2407.44 & 1481.62 & 1478.40 \\
\hline AIC & 4933.68 & 4933.74 & 2417.54 & 2417.44 & 1489.62 & 1488.40 \\
\hline $\mathrm{BIC}$ & 4959.69 & 4966.25 & 2440.69 & 2446.37 & 1510.82 & 1514.89 \\
\hline
\end{tabular}

$\sigma_{\mathrm{a}}^{2}$ : direct additive effect; $\sigma_{\mathrm{m}}^{2}$ : maternal additive genetic variance; $\sigma_{\mathrm{pe}}^{2}:$ maternal permanent environmental variance; $\sigma_{\mathrm{ce}}^{2}$ : common environmental variance; $\sigma_{\mathrm{cy}}^{2}$ : cytoplasmic genetic variance; $\sigma_{\mathrm{e}}^{2}$ : error variance; $\sigma_{\mathrm{p}}^{2}$ : phenotypic variance; $\mathrm{h}^{2}$ : direct heritability; $\mathrm{m}^{2}$ : maternal heritability; $\mathrm{pe}^{2}$ : maternal permanent environmental variance expressed as a proportion of phenotypic variance; $\mathrm{ce}^{2}$ : maternal common environmental variance expressed as a proportion of the phenotypic variance; $\mathrm{cy}^{2}$ : cytoplasmic genetic variance expressed as a proportion of phenotypic variance; $-2 * \log L$ : $\log$ likelihood ratio test: AIC: Akaike Information Criterion; BIC: Bayesian Information Criterion. Value in bold shows the chosen model according to test statistic.

maternal permanent environmental and common environmental effects were important components for the estimation of genetic parameters with the exception that the maternal permanent environmental effect was not significant for fat depth. Maternal heritability estimates obtained from the full dataset were 0.06, 0.05, 0.02 and 0.05 for EWW, SW, MD and FD respectively. The maternal common environmental effect was the largest maternal effect contributing to phenotypic variance, and in the full dataset ranged from 0.09 to 0.24 across the four traits.

There were no significant differences in $\log$ likelihood $(\log L)$ values between models that did and did not include cytoplasmic effects, and variance components were virtually the same in both models for EWW and MD. 
$\operatorname{LogL}$ values for SW and FD increased slightly with the inclusion of cytoplasmic effects, but in only one dataset (for SW and FD) was the difference significant. For both SW and FD, cytoplasmic effects accounted for approximately $1-2 \%$ of the phenotypic variation. The phenotypic variances and residual errors remained approximately the same in both models, which meant that, for SW and FD, the inclusion of cytoplasmic effects caused slight decreases in direct heritability and maternal heritability.

It was observed that some maternal lines in the dataset had very few animals. Inclusion of such lines in an analysis may cause maternal lineage variance to be confounded with the direct additive genetic effect. Maternal lines with few animals could result from a lack of pedigree information. In the study of Roughsedge et al. [13] of dairy cows, the authors commented that some cows could not be traced back to a distant cytoplasmic origin, and might be of the same maternal lineage as others in the dataset. If there is insufficient information, maternal subfamilies can be assigned to different maternal lines, although they may in fact belong to one true family. Roughsedge et al. [14] concluded from a simulation study that incomplete maternal pedigree information, resulting in animals being assigned to different lineages when they actually belong to the same one, could cause a downward bias in estimates of cytoplasmic effect.

Some authors have commented that analyses on small datasets from a limited number of populations may reduce the ability of tests to find significant differences between maternal lines $[9,12,15,16]$. Mezzadra et al. [12] examined the influence of cytoplasmic inheritance by a method of assigning maternal lines, in addition to analysing the direct association between mitochondrial DNA polymorphisms and productive traits. It was found that in 23 supposed maternal lineages there were only five different mitochondrial genotypes, and it was suggested that the failure to detect a cytoplasmic effect could be a reflection of some maternal lines having the same genotype. Eledath and Hines [4] studied Holstein cattle and found ten different mitochondrial genotypes in 16 maternal lines. In the study described in this chapter, data came from three Welsh Mountain breeding groups, in which there might be a greater chance of finding variation among maternal lines than in a closed flock. However, as with other studies, there is always the possibility that animals assigned to different lines within flocks could be subfamilies sharing a common mitochondrial genome.

Roughsedge et al. [13] found no significant differences among maternal lineages for yield traits in dairy cattle. However, when maternal lineages were restricted to those with five or more records, maternal lineage contributed 4.4\% of phenotypic variance for persistency of milk production. Boettcher et al. [3] 
also suggested that more accurate estimates of maternal lineage effects would be obtained with more animals per maternal line. In this study, it was observed for both SW and FD that the contribution made by maternal line to phenotypic variance increased since maternal lines were restricted to those with higher numbers of animals. For SW the contribution due to cytoplasmic effect was $1.4 \%$ of phenotypic variance in the dataset with all animals, and increased to $1.9 \%$ when the dataset was restricted to maternal lines with 20 or more animals per line. Similarly, for FD the contribution to phenotypic variance increased from $1.3 \%$ to $2.2 \%$ when these datasets were compared.

Methods based on detecting mitochondrial DNA polymorphisms are probably more accurate than those based on assigning individuals to maternal lines, given the inaccuracies that arise in determining maternal pedigree. It should be noted, however that the same mitochondrial genotype can occur in different families. It was pointed out by Schnitzenlehner and Essl [15] that treating animals as members of a maternal line to have the same mitochondrial genome can become more unreliable with increasing numbers of generations, since the probability of mutation increases with increasing distance from the foundation dam.

Studies that have examined mitochondrial DNA polymorphism have also found that some are associated with certain traits. Mannen et al. [10] studied the association between the variation of transcribed mtDNA and variation in carcass traits in beef cattle, and it was suggested that one polymorphism was associated with meat quality traits, the longissimus muscle area and beef marbling score. Schnitzenlehner and Essl [15] found that cytoplasmic effects were non-significant for dairy traits, but significant for fitness traits in cattle. Nevertheless, even if associations between mitochondrial polymorphisms and economically important traits exist, Sutarno et al. [20] commented that mitochondrial markers were less valuable than nuclear ones in marker-assisted selection because they are maternally inherited, and because selection intensities in females are much less than in males, and producing far fewer progeny than males.

In previous studies in sheep, it has been concluded that cytoplasmic influences were not required in models estimating genetic parameters for the same growth and carcass traits studied here. The results suggest some mitochondrial influence on traits related to body composition, although such effects could not be demonstrated conclusively with the methods or datasets available. It is possible that the impacts of such effects are difficult to determine by standard quantitative methods. Perhaps the models are not able to distinguish between them and other maternal effects and this would also depend upon the data 
structure. The results of this study show that cytoplasmic effects were generally not significant for the four traits examined. Therefore, in agreement with other studies of sheep, beef and dairy cattle and assuming that these results are equally applicable to other sheep populations the inclusion of cytoplasmic effect in models to estimate genetic parameters is not necessarily required. Further work could include looking at the effect of the number of generations in the dataset.

\section{ACKNOWLEDGEMENTS}

Many thanks to Geoff Pollott, the Welsh Mountain breeding groups CAMDA, CAMP and Llysfasi, and the Meat and Livestock Commission.

\section{REFERENCES}

[1] Abney M., McPeek M.S., Ober C., Estimation of variance components of quantitative traits in inbred populations, Am. J. Hum. Genet. 66 (2000) 629-650.

[2] Ap Dewi I., Saatci M., Ulutas Z., Genetic parameters of weights, ultrasonic muscle and fat depths, maternal effects and reproductive traits in Welsh Mountain sheep, Anim. Sci. 74 (2002) 399-408.

[3] Boettcher P.J., Steverink D.W.B., Beitz D.C., Freeman A.E., McDaniel B.T., Multiple herd evaluation of the effects of maternal lineage on yield traits of Holstein cows, J. Dairy Sci. 79 (1996) 655-662.

[4] Eledath F.M., Hines H.C., Detection of nucleotide variations in the D-loop of bovine mitochondrial DNA using polymerase chain reaction-based methodologies, Anim. Genet. 27 (1996) 333-336.

[5] Gilmour A.R., Gogel B.J., Cullis B.R., Welham S.J., Thompson R., ASReml User Guide Release 1.0. VSN International Ltd, Hemel Hempstead, HP1 1ES, UK, 2002.

[6] Hanford K.J., Snowder G.D., van Vleck L.D., Models with nuclear, cytoplasmic, and environmental effects for production traits of Columbia sheep, J. Anim. Sci. 81 (2003) 1926-1932.

[7] Hayashida K., Omagari K., Masuda J., Hazama H., Kadokawa Y., Ohba K., Kohno S., The sperm mitochondria-specific translocator has a key role in maternal mitochondrial inheritance, Cell Biol. Int. 29 (2005) 472-481.

[8] Maniatis N., Pollott G.E., Nuclear, cytoplasmic, and environmental effects on growth, fat, and muscle traits in Suffolk lambs from a sire referencing scheme, J. Anim. Sci. 80 (2002) 57-67.

[9] Mannen H., Kojima T., Oyama K., Makai F., Ishida T., Tsuji S., Effect of mitochondrial DNA variation on carcass traits of Japanese Black cattle, J. Anim. Sci. 76 (1998) 36-41. 
[10] Mannen H., Morimoto M., Oyama K., Makai F., Tsuji S., Identification of mitochondrial DNA substitution related to meat quality in Japanese Black cattle, J. Anim. Sci. 81 (2003) 68-73.

[11] Meyer K., Variance components due to direct and maternal effects for growth traits of Australian beef cattle, Livest. Prod. Sci. 31 (1992) 179-204.

[12] Mezzadra C.A., Melucci L.M., Corva P.M., Valiente S.L., Rípoli M.V., Lirón P., Giovambattista G., Effects of cytoplasmic inheritance on preweaning traits of Hereford cattle, Genet. Mol. Biol. 28 (2005) 357-362.

[13] Roughsedge T., Visscher P.M., Brotherstone S., Effects of cow families on production traits in dairy cattle, Anim. Sci. 71 (2000) 49-57.

[14] Roughsedge T., Brotherstone S., Visscher P.M., Bias and power in the estimation of a maternal family variance component in the presence of incomplete and incorrect pedigree information, J. Dairy Sci. 84 (2001) 944-950.

[15] Schnitzenlehner S., Essl A., Field data analysis of cytoplasmic inheritance of dairy and fitness-related traits in cattle, Anim. Sci. 68 (1999) 459-466.

[16] Schutz M.M., Freeman A.E., Lindberg G.L., Kochler C.M., Beitz D.C., The effect of mitochondrial DNA on milk production and health of dairy cattle, Livest. Prod. Sci. 37 (1994) 283-295.

[17] Schwarz G., Estimating the dimension of a model, Ann. Statist. 6 (1978) 461-464.

[18] Signet, Recording protocols: Practical requirements for sheep scanning, http://www.signetfbc.co.uk [consulted 8 August 2007].

[19] Snowder G.D., Hanford K.J., van Vleck L.D., Comparison of models including cytoplasmic effects for traits of Rambouillet sheep, Livest. Prod. Sci. 90 (2004) 159-166.

[20] Sutarno, Cummins J.M., Greeff J., Lymbery A.J., Mitochondrial DNA polymorphisms and fertility in beef cattle, Theriogenology 57 (2002) 1603-1610.

[21] Tess M.W., Robison O.W., Evaluation of cytoplasmic genetic effects in beef cattle using an animal model, J. Anim. Sci. 68 (1990) 1899-1909.

[22] van Vleck LD., Snowder GD., Hanford KJ., Models with cytoplasmic effects for birth, weaning, and fleece weights, and litter size at birth for a population of Targhee sheep, J. Anim. Sci. 81 (2003) 61-67.

[23] van Vleck L.D., Hanford K.J., Snowder G.D., Lack of evidence for cytoplasmic effects for four traits of Polpay sheep, J. Anim. Sci. 83 (2005) 552-556.

[24] Wada Y., Kashiwagi N., Selecting statistical models with information statistics, J. Dairy Sci. 73 (1990) 3575-3582.

[25] Wagner R.P., The role of maternal effects in animal breeding: II. Mitochondria and animal inheritance, J. Anim. Sci. 35 (1972) 1280-1287.

[26] Zhao X., Li N., Guo W., Hu X., Liu Z., Gong G., Wang A., Feng J., Wu C., Further evidence for paternal inheritance of mitochondrial DNA in sheep (Ovis aries), Heredity 93 (2004) 399-403. 